\title{
SECOND HANKEL DETERMINANT FOR A CLASS OF ANALYTIC FUNCTIONS DEFINED BY A LINEAR OPERATOR
}

\author{
AABED MOHAMMED AND MASLINA DARUS
}

\begin{abstract}
By making use of the linear operator $\Theta_{m}^{\lambda, n}, m \in \mathbb{N}=\{1,2,3, \ldots\}$ and $\lambda, n \in \mathbb{N}_{0}=$ $\mathbb{N} \cup\{0\}$ given by the authors, a class of analytic functions $S_{m}^{\lambda, n}(\alpha, \sigma)(|\alpha|<\pi / 2,0 \leq \sigma<1)$ is introduced. The object of the present paper is to obtain sharp upper bound for functional $\left|a_{2} a_{4}-a_{3}^{2}\right|$.
\end{abstract}

\section{Introduction}

Let $\mathscr{A}$ denote the class of normalised analytic functions of the form

$$
f(z)=z+\sum_{k=2}^{\infty} a_{k} z^{k}
$$

where $z \in U:=\{z: z \in \mathbb{C}$ and $|z|<1\}$. Let $S$ denote the class of all functions in $\mathscr{A}$ which are univalent.

Robertson [14] introduced the class of starlike functions of order $\sigma$ as follows:

Definition $1.1([14])$. Let $\sigma \in[0,1], f \in S$ and

$$
\Re\left\{\frac{z f^{\prime}(z)}{f(z)}\right\}>\sigma, \quad z \in U .
$$

Then, we say that $f$ is a starlike function of order $\sigma$ on $U$ and we denoted this class by $S^{*}(\sigma)$.

Spacek [15] introduced the class of spirallike functions of type $\alpha$ as follows:

Theorem 1.1 ([15]). Let $f \in S$ and $-\pi / 2<\alpha<\pi / 2$. Then $f(z)$ is a spirallike function of type $\alpha$ on $U$ if

$$
\Re\left\{e^{i \alpha} \frac{z f^{\prime}(z)}{f(z)}\right\}>0, \quad z \in U
$$

We denoted this class by $S_{\alpha}$.

Corresponding author: Maslina Darus.

2010 Mathematics Subject Classification. 30C45.

Key words and phrases. Hankel determinant, Positive real functions, Linear operator. 
From Definition 1.1 and Theorem 1.1, it is easy to see ([17])that starlike functions of order $\sigma$ and spirallike functions of type $\alpha$ have some relationships on geometry. Starlike functions of order $\sigma$ map $U$ into the right half complex plane whose real part is greater than $\sigma$ by the mapping $\frac{z f^{\prime}(z)}{f(z)}$, while spirallike functions of type $\alpha$ map $U$ in to the right half complex plane by the mapping $e^{i \alpha} \frac{z f^{\prime}(z)}{f(z)}$. Since $\lim _{z \rightarrow 0} e^{i \alpha} \frac{z f^{\prime}(z)}{f(z)}=e^{i \alpha}$, we can deduce that if we restrict the image of the mapping $e^{i \alpha} \frac{z f^{\prime}(z)}{f(z)}$ in the right complex plane whose real part is greater than a certain constant, then the constant must be smaller than $\cos \alpha$.

Libera [16] introduced and studied the class $S_{\sigma}^{\alpha}$ given as follows:

Definition 1.2 ([16]). Let $\sigma \in\left[0,1\left[,-\pi / 2<\alpha<\pi / 2\right.\right.$ and $f \in S$. Then $f \in S_{\sigma}^{\alpha}$ if and only if

$$
\Re\left\{e^{i \alpha} \frac{z f^{\prime}(z)}{f(z)}\right\}>\sigma \cos \alpha, \quad z \in U .
$$

Obviously,

$$
S_{\sigma}^{0}=S^{*}(\sigma) \text { and } S_{0}^{\alpha}=S_{\alpha}
$$

For $f_{j} \in \mathscr{A}$ given by

$$
f_{j}(z)=z+\sum_{k=2}^{\infty} a_{k, j} z^{k}(j=1,2),
$$

the Hadamard product (or convolution) $f_{1} * f_{2}$ of $f_{1}$ and $f_{2}$ is defined by

$$
\left(f_{1} * f_{2}\right)(z)=z+\sum_{k=2}^{\infty} a_{k, 1} a_{k, 2} z^{k}(z \in U) .
$$

We recall that a family of the Hurwitz-Lerch Zeta functions $\Phi_{\mu, v}^{(\rho, \sigma)}(z, s, a)$ ([12]) is defined by

$$
\begin{gathered}
\Phi_{\mu, v}^{(\rho, \sigma)}(z, s, a)=\sum_{n=0}^{\infty} \frac{(\mu)_{\rho n}}{(v)_{\sigma n}} \frac{z^{n}}{(n+a)^{s}}, \\
\left(\mu \in \mathbb{C} ; a, v \in \mathbb{C} \backslash \mathbb{Z}_{0}^{-} ; \rho, \sigma \in \mathbb{R}^{+}, \rho<\sigma \text { when } s, z \in \mathbb{C} ;\right. \\
\rho=\sigma \text { and } s \in \mathbb{C} \text { when }|z|<1 ; \rho=\sigma \text { and } \\
\Re(s-\mu+v)>1 \text { when }|z|=1),
\end{gathered}
$$

contains as its special cases, not only the Hurwitz-Lerch Zeta function

$$
\Phi_{\mu, v}^{(\rho, \sigma)}(z, s, a)=\Phi_{\mu, v}^{(0,0)}(z, s, a)=\sum_{n=0}^{\infty} \frac{z^{n}}{(n+a)^{s}},
$$

but also the following generalized Hurwitz-Zeta function introduced and studied earlier by Goyal and Laddha ([13]),

$$
\Phi_{\mu, 1}^{(1,1)}(z, s, a)=\Phi_{\mu}(z, s, a)=\sum_{n=0}^{\infty} \frac{(\mu)_{n}}{n !} \frac{z^{n}}{(n+a)^{s}},
$$


which, for convenience, are called the Goyal-Laddha-Hurwitz-Lerch Zeta function. Here $(x)_{k}$ is Pochhammer symbol (or the shifted factorial, since $(1)_{k}=k !$ ) and $(x)_{k}$ given in terms of the Gamma functions can be written as

$$
(x)_{k}=\frac{\Gamma(x+k)}{\Gamma(x)} \begin{cases}1, & \text { if } k=0 \text { and } x \in \mathbb{C} \backslash\{0\} \\ x(x+1) \ldots(x+k-1), & \text { if } k \in \mathbb{N} \text { and } x \in \mathbb{C} .\end{cases}
$$

It follows that the authors [1] introduced the linear operator $\Theta_{m}^{\lambda, n} f(z)$ as the following. For $a=1$, in (1.2), we consider the function

$$
G(z)=z \Phi_{\mu}(z, s, 1)=z+\sum_{k=2}^{\infty} \frac{(\mu)_{k-1}}{(k-1) !} \frac{z^{k}}{k^{s}} .
$$

Thus

$$
\begin{gathered}
G(z) * G(z)^{(-1)}=\frac{z}{(1-z)^{\lambda+1}}, \quad \lambda>-1 \\
=z+\sum_{k=2}^{\infty} \frac{(\lambda+1)_{k-1}}{(k-1) !} z^{k}
\end{gathered}
$$

Now for $s=n, \lambda \in \mathbb{N}_{0}$ and $\mu=m \in \mathbb{N}$, we define the linear operator

$$
\begin{aligned}
\Theta_{m}^{\lambda, n} f(z) & =G(z)^{(-1)} * f(z) . \quad(f \in \mathscr{A}) \\
& =z+\sum_{k=2}^{\infty} \frac{(\lambda+1)_{k-1}}{(m)_{k-1}} k^{n} a_{k} z^{k} .
\end{aligned}
$$

In [10], Noonan and Thomas stated that the $q$ th Hankel determinant of the function $f$ of the form (1.1) is defined for $q \in \mathbb{N}$ by

$$
H_{q}(k)=\left|\begin{array}{cccc}
a_{k} & a_{k+1} & \cdots & a_{k+q+1} \\
a_{k+1} & a_{k+2} & \cdots & a_{k+q+2} \\
\vdots & \vdots & \vdots & \vdots \\
a_{k+q-1} & a_{k+q} & \cdots & a_{k+2 q-2}
\end{array}\right| .
$$

We now introduce the following class of functions.

Definition 1.3. The function $f \in \mathscr{A}$ is said to be in the class $S_{m}^{\lambda, n}(\alpha, \sigma),(|\alpha|<\pi / 2,0 \leq \sigma<1)$ if it satisfies the inequality

$$
\Re\left\{e^{i \alpha} \frac{\Theta_{m}^{\lambda, n} f(z)}{z}\right\}>\sigma \cos \alpha \quad(z \in U)
$$

As is usually the case, we let $P$ be the family of all functions $p$ analytic in $U$ for which $\Re\{p(z)\}>0$ and

$$
p(z)=1+c_{1} z+c_{2} z^{2}+\ldots, \quad z \in U
$$


It follows from (1.4) that

$$
f \in S_{m}^{\lambda, n}(\alpha, \sigma) \Leftrightarrow e^{i \alpha} \frac{\Theta_{m}^{\lambda, n} f(z)}{z}=[(1-\sigma) p(z)+\sigma] \cos \alpha+i \sin \alpha,
$$

where $\alpha$ is real, $|\alpha|<\pi / 2$ and $p(z) \in P$.

We note that

$$
\begin{aligned}
& S_{1}^{0,0}(\alpha, \sigma)=\left\{f: f \in \mathscr{A} \text { and } \Re\left\{e^{i \alpha} \frac{f(z)}{z}\right\}>\sigma \cos \alpha\right\}, \\
& S_{1}^{0,1}(\alpha, \sigma)=\left\{f: f \in \mathscr{A} \text { and } \Re\left\{e^{i \alpha} f^{\prime}(z)\right\}>\sigma \cos \alpha\right\}, \\
& S_{1}^{0,1}(0,0)=S_{1}^{1,0}(0,0)=S_{2}^{1,1}(0,0)=\mathscr{R}:=\left\{f: f \in \mathscr{A} \text { and } \Re\left\{f^{\prime}(z)\right\}>0\right\} .
\end{aligned}
$$

Remark 1.1 ([6]). The subclass $\mathscr{R}$ was studied systematically by MacGregor ([11]) who indeed referred to numerous earlier investigations involving functions whose derivative has a positive real part.

It is well known ([9]) that for $f \in S$ and given by (1.1) the sharp inequality $\left|a_{3}-a_{2}^{2}\right| \leq 1$ holds. This corresponds to the Hankel determinant with $q=2$ and $k=1$. For a given family $\mathscr{F}$ of functions in $\mathscr{A}$, the sharp bound for the nonlinear functional $\left|a_{2} a_{4}-a_{3}^{2}\right|$ is popularly known as the second Hankel determinant. This corresponds to the Hankel determinant with $q=2$ and $k=2$. The second Hankel determinant for some subclasses of analytic and nuivalent functions has been studied by many authors (see [2]-[6], [18], [19]).

In the present paper, we seek upper bound for the functional $\left|a_{2} a_{4}-a_{3}^{2}\right|\left(f \in S_{m}^{\lambda, n}(\alpha, \sigma)\right)$. Our investigation includes a recent result of Janteng et al. [2].

To prove our main result, we need the following lemmas.

Lemma 1.2 ([9]). Let the function $p \in P$ and be given by the series (1.5). Then, the sharp estimate

$$
\left|c_{k}\right| \leq 2(k \in \mathbb{N})
$$

holds.

Lemma 1.3 ([7] and [8]). Let the function $p \in P$ be given by the series (1.5). Then

$$
2 c_{2}=c_{1}^{2}+x\left(4-c_{1}^{2}\right)
$$

for some $x,|x| \leq 1$ and

$$
4 c_{3}=c_{1}^{3}+2\left(4-c_{1}^{2}\right) c_{1} x-c_{1}\left(4-c_{1}^{2}\right) x^{2}+2\left(4-c_{1}^{2}\right)\left(1-|x|^{2}\right) z
$$

for some $z,|z| \leq 1$. 


\section{Main results}

We prove the following.

Theorem 2.1. Let the function $f$ given by (1.1) be in the class $S_{m}^{\lambda, n}(\alpha, \sigma)$. Then

$$
\left|a_{2} a_{4}-a_{3}^{2}\right| \leq \frac{4 m^{2}(1-\sigma)^{2}(1+m)^{2} \cos ^{2} \alpha}{3^{2 n}(\lambda+1)^{2}(\lambda+2)^{2}} .
$$

The estimate (2.1) is sharp.

Proof. Let $f \in S_{m}^{\lambda, n}(\alpha, \sigma)$. Then from (1.6)we have

$$
e^{i \alpha} \frac{\Theta_{m}^{\lambda, n} f(z)}{z}=[(1-\sigma) p(z)+\sigma] \cos \alpha+i \sin \alpha,
$$

where $p \in P$ and is given by (1.5). Then

$$
e^{i \alpha}\left\{1+\sum_{k=2}^{\infty} \frac{(k+\lambda-1) !(m-1) !}{\lambda !(k+m-2) !} k^{n} a_{k} z^{k-1}\right\}=\left[(1-\sigma)\left(1+\sum_{k=1}^{\infty} c_{k} z^{k}\right)+\sigma\right] \cos \alpha+i \sin \alpha .
$$

Comparing the coefficients, we get

$$
\left.\begin{array}{l}
\frac{(\lambda+1)}{m} 2^{n} e^{i \alpha} a_{2}=(1-\sigma) c_{1} \cos \alpha \\
\frac{(\lambda+2)(\lambda+1)}{m(m+1)} 3^{n} e^{i \alpha} a_{3}=(1-\sigma) c_{2} \cos \alpha \\
\frac{(\lambda+3)(\lambda+2)(\lambda+1)}{m(m+1)(m+2)} 4^{n} e^{i \alpha} a_{4}=(1-\sigma) c_{3} \cos \alpha
\end{array}\right\}
$$

Therefore, (2.2) yields

$$
\left|a_{2} a_{4}-a_{3}^{2}\right|=\frac{m^{2}(1-\sigma)^{2}(1+m) \cos ^{2} \alpha}{(\lambda+1)^{2}(\lambda+2)}\left|\frac{(m+2) c_{1} c_{3}}{2^{3 n}(\lambda+3)}-\frac{c_{2}^{2}(m+1)}{3^{2 n}(\lambda+2)}\right| .
$$

Since the functions $p(z)$ and $p\left(e^{i \theta} z\right),(\theta \in \mathbb{R})$ are members of the class $P$ simultaneously, we assume without loss of generality that $c_{1}>0$. For convenience of notation, we take $c_{1}=c, c \in$ [0,2]. Using (1.7) along with (1.8), we get

$$
\begin{aligned}
\left|a_{2} a_{4}-a_{3}^{2}\right|= & \frac{m^{2}(1-\sigma)^{2}(1+m) \cos ^{2} \alpha}{4(\lambda+1)^{2}(\lambda+2)}\left\{\mid \frac{(m+2)}{2^{3 n}(\lambda+3)}\left[c^{4}+2 c^{2}\left(4-c^{2}\right) x-c^{2}\left(4-c^{2}\right) x^{2}\right.\right. \\
& \left.\left.+2 c\left(4-c^{2}\right)\left(1-|x|^{2}\right) z\right]-\frac{(m+1)}{3^{2 n}(\lambda+2)}\left[c^{4}+2 c^{2}\left(4-c^{2}\right) x+x^{2}\left(4-c^{2}\right)^{2}\right] \mid\right\} \\
= & \frac{m^{2}(1-\sigma)^{2}(1+m) \cos ^{2} \alpha}{4(\lambda+1)^{2}(\lambda+2)} \mid\left\{\frac{(m+2)}{2^{3 n}(\lambda+3)}-\frac{(m+1)}{3^{2 n}(\lambda+2)}\right\} c^{4} \\
& +\left\{\frac{(m+2)}{2^{3 n}(\lambda+3)}-\frac{(m+1)}{3^{2 n}(\lambda+2)}\right\} 2 c^{2}\left(4-c^{2}\right) x
\end{aligned}
$$




$$
-\left\{\frac{c^{2}(m+2)}{2^{3 n}(\lambda+3)}+\frac{(m+1)\left(4-c^{2}\right)}{3^{2 n}(\lambda+2)}\right\} x^{2}\left(4-c^{2}\right)+\frac{2(m+2)}{2^{3 n}(\lambda+3)} c\left(4-c^{2}\right)\left(1-|x|^{2}\right) z \mid .
$$

An application of triangle inequality and replacement of $|x|$ by $y$ give

$$
\begin{aligned}
\left|a_{2} a_{4}-a_{3}^{2}\right| \leq & \frac{m^{2}(1-\sigma)^{2}(1+m) \cos ^{2} \alpha}{4(\lambda+1)^{2}(\lambda+2)}\left[\left\{\frac{(m+2)}{2^{3 n}(\lambda+3)}-\frac{(m+1)}{3^{2 n}(\lambda+2)}\right\} c^{4}\right. \\
& +\left\{\frac{(m+2)}{2^{3 n}(\lambda+3)}-\frac{(m+1)}{3^{2 n}(\lambda+2)}\right\} 2 c^{2} y\left(4-c^{2}\right) \\
& \left.+\left\{\frac{c^{2}(m+2)}{2^{3 n}(\lambda+3)}+\frac{(m+1)\left(4-c^{2}\right)}{3^{2 n}(\lambda+2)}\right\} y^{2}\left(4-c^{2}\right)+\frac{2(m+2)}{2^{3 n}(\lambda+3)} c\left(4-c^{2}\right)\left(1-y^{2}\right)\right] \\
= & \frac{m^{2}(1-\sigma)^{2}(1+m) \cos ^{2} \alpha}{4(\lambda+1)^{2}(\lambda+2)}\left[\left\{\frac{(m+2)}{2^{3 n}(\lambda+3)}-\frac{(m+1)}{3^{2 n}(\lambda+2)}\right\} c^{4}\right. \\
& +\left\{\frac{(m+2)}{2^{3 n}(\lambda+3)}-\frac{(m+1)}{3^{2 n}(\lambda+2)}\right\} 2 c^{2} y\left(4-c^{2}\right) \\
& \left.+\left\{\frac{c(c-2)(m+2)}{2^{3 n}(\lambda+3)}+\frac{(m+1)\left(4-c^{2}\right)}{3^{2 n}(\lambda+2)}\right\} y^{2}\left(4-c^{2}\right)+\frac{2(m+2)}{2^{3 n}(\lambda+3)} c\left(4-c^{2}\right)\right] \\
= & G(c, y), \quad 0 \leq c \leq 2 \text { and } 0 \leq y \leq 1 .
\end{aligned}
$$

We next maximize the function $G(c, y)$ on the closed square $[0,2] \times[0,1]$. Since

$$
\begin{aligned}
\frac{\partial G}{\partial y}= & \frac{m^{2}(1-\sigma)^{2}(1+m) \cos ^{2} \alpha}{4(\lambda+1)^{2}(\lambda+2)}\left[\left\{\frac{(m+2)}{2^{3 n}(\lambda+3)}-\frac{(m+1)}{3^{2 n}(\lambda+2)}\right\} 2 c^{2}\left(4-c^{2}\right)\right. \\
& \left.+\left\{\frac{c(c-2)(m+2)}{2^{3 n}(\lambda+3)}+\frac{(m+1)\left(4-c^{2}\right)}{3^{2 n}(\lambda+2)}\right\} 2 y\left(4-c^{2}\right)\right],
\end{aligned}
$$

$c-2<0,3^{2 n}(m+2)(\lambda+2)>2^{3 n}(m+1)(\lambda+3)$, we have $\partial G / \partial y>0$ for $0<c<2,0<y<1$. Thus $G(c, y)$ cannot have a maximum in the interior of the closed square $[0,2] \times[0,1]$. Moreover, for fixed $c \in[0,2], \max _{0 \leq y \leq 1} G(c, y)=G(c, 1)=F(c)$. Since

$$
\begin{aligned}
F(c)= & \frac{m^{2}(1-\sigma)^{2}(1+m) \cos ^{2} \alpha}{4(\lambda+1)^{2}(\lambda+2)}\left[\left\{\frac{(m+2)}{2^{3 n}(\lambda+3)}-\frac{(m+1)}{3^{2 n}(\lambda+2)}\right\} c^{4}\right. \\
& +\left\{\frac{(m+2)}{2^{3 n}(\lambda+3)}-\frac{(m+1)}{3^{2 n}(\lambda+2)}\right\} 2 c^{2}\left(4-c^{2}\right) \\
& \left.+\left\{\frac{c^{2}(m+2)}{2^{3 n}(\lambda+3)}+\frac{(m+1)\left(4-c^{2}\right)}{3^{2 n}(\lambda+2)}\right\}\left(4-c^{2}\right)\right]
\end{aligned}
$$

Then

$$
F^{\prime}(c)=\frac{2 m^{2}(1-\sigma)^{2}(1+m) \cos ^{2} \alpha}{(\lambda+1)^{2}(\lambda+2)}\left\{\frac{c\left(3-c^{2}\right)(m+2)}{2^{3 n}(\lambda+3)}-\frac{c\left(4-c^{2}\right)(m+1)}{3^{2 n}(\lambda+2)}\right\},
$$

so that $F^{\prime}(c)<0$ for $0<c<2$ and has real critical point at $c=0$. Also $F(c)>F(2)$. Therefore, $\max _{0 \leq c \leq 2} F(c)$ occurs at $c=0$. Therefore, the upper bound of (2.3) corresponds to $y=1, c=0$. Hence

$$
\left|a_{2} a_{4}-a_{3}^{2}\right| \leq \frac{4 m^{2}(1-\sigma)^{2}(1+m)^{2} \cos ^{2} \alpha}{3^{2 n}(\lambda+1)^{2}(\lambda+2)^{2}} .
$$


which is the assertion (2.1).Equality holds for the function

$$
f(z)=\left(\sum_{k=1}^{\infty} \frac{(m)_{k-1}}{(\lambda+1)_{k-1} k^{n}} z^{k}\right) * e^{-i \alpha}\left[z\left(\frac{1+(1-2 \sigma) z^{2}}{1-z^{2}} \cos \alpha+i \sin \alpha\right)\right] .
$$

This completes the proof of the Theorem 2.1.

Remark 2.1. For $\alpha=0, \sigma=0, \lambda=m=1, n=0$ and for $\alpha=0, \sigma=0, \lambda=1, m=2, n=1$ we get a resent result due to Janteng et al. [2] as in the following corollary.

Corollary 2.1. If $f \in \mathscr{R}$ then

$$
\left|a_{2} a_{4}-a_{3}^{2}\right| \leq \frac{4}{9}
$$

The result is sharp.

\section{Acknowledgement}

The work here is fully supported by MOHE Grant: UKM-ST-06-FRGS0244-2010, Malaysia.

\section{References}

[1] A. Mohammed and M. Darus, An operator defined by convolution involving the generalized Hurwitz-Lerch zeta function, Submitted

[2] A. Janteng, S. A. Halim, and M. Darus, Coefficient inequality for a function whose derivative has positive real part, J.Ineq. Pure and Appl. Math.,7(2)(2006), 1-5.

[3] A. Janteng, S. A. Halim, and M. Darus, Hankel determinant for starlike and convex functions, Int. Journal of Math. Analysis, 1(13)(2007), 619 - 625.

[4] A. K. Mishra and P. Gochhayat, Second Hankel determinant for a class of ana lytic functions defined by fractional derivative functions, International Journal of Mathematics and Mathematical Sciences, 2008, Article ID 153280, 10 pages.

[5] S. C. Soh and D. Mohamad, Coefficient bounds for certain classes of close-to-convex functions, Int. Journal of Math. Analysis, 2(27)(2008), 1343 - 1351.

[6] G. Murugusundaramoorthy and N. Magesh, Coefficient inequalities for certain classes of analytic functions associated with Hankel determinant, Bulletin of Math. Anal. Appl., 1(3)(2009),85-89.

[7] R. J. Libera and E. J. Zlotkiewicz, Early coefficients of the inverse of a regular convex function, Proc. Amer. Math. Soc., 85(2)(1982), 225-230.

[8] R. J. Libera and E. J. Zlotkiewicz, Coeffcient bounds for the inverse of a function with derivative in P, Proc. Amer. Math. Soc., 87(2)(1983), 251-289.

[9] P. L. Duren, Univalent Functions, Springer-Verlag, New York, Berlin, Heidelberg, Tokyo, 1983.

[10] J. W. Noonan and D.K.Thomas, On the second Hankel determinant of areally mean p--valent functions, Trans. Amer. Math. Soc., 223(2)(1976), 337-346.

[11] T. H. MacGregor,Functions whose derivative has a positive real part, Trans. Amer. Math. Soc., 104(1962), 532537.

[12] S. D. Lin, H. M.Srivastava, Some families of the Hurwitz-Lerch Zeta function and associated fractional derivative and other integral representations, Appl. Math. Comput., 154(2004), 725-733.

[13] S. Goyal, R. K. Laddha, On the generalized Riemann zeta function and the generalized Lambert transform, Ganita Sandesh, 11(1997), 99-108. 
[14] M.S. Robertson, On the theory of univalent functions, Ann. Math., 37(1936), 374-408.

[15] L. Spacek, Contribution la thorie des fonctions univalentes, Casopis Pest Math., 62(1932), 1219, (in Russian).

[16] J. R. Libera, Univalent $\alpha$ - - spiral functions, Canada. J. Math., 19(1967), 449-456.

[17] Q. Xu and S. Lu, The Alexander transformation of a subclass of spirallike functions of type $\beta$, J. Ineq. Pure and Appl. Math, 10(1)(2009), 1-7.

[18] T. Hayami and S. Owa, Hankel determinant for p-valently starlike and convex functions of order $\alpha$, General Math., 17 (2009), 29-44.

[19] T. Hayami and S. Owa, Generalized Hankel determinant for certain classes, Int. J. Math. Anal., 4 (2010), 25732585.

School of Mathematical Sceinces, Faculty of Science and Technology, Universiti Kebangsaan Malaysia 43600 Bangi, Selangor D. Ehsan, Malaysia.

E-mail: aabedukm@yahoo.com

School of Mathematical Sceinces, Faculty of Science and Technology, Universiti Kebangsaan Malaysia 43600 Bangi, Selangor D. Ehsan, Malaysia.

E-mail: maslina@ukm.my 